\title{
Broadening horizons: Integrating quantitative and qualitative research
}

$\mathrm{H}$ ealth research usually employs quantitative, often experimental, methods to study clinical conditions and outcomes. The use of qualitative methods in this type of research is much less common. However, we contend that qualitative research, in combination with quantitative research, can play an important role generating an improved understanding of disease, health and health care.

Data collected in qualitative research are usually in narrative rather than numerical form, such as the transcript of an unstructured, in-depth interview. Analysis of qualitative data organizes, summarizes and interprets these nonnumerical observations. The goal of qualitative research is the development of concepts that help clarify phenomena in natural, rather than experimental, settings, giving due emphasis to the meanings, experiences and views of all the participants being studied. For example, to understand why some members of ethnic minorities have refused tuberculosis treatment, qualitative, culturally sensitive interviews may be much more informative than standardized quantitative interviews.

Both quantitative and qualitative research have weaknesses that to some degree are compensated for by the strengths of the other. Quantitative research is very well suited to establishing cause-and-effect relationships, to testing hypotheses and to determining the opinions, attitudes and practices of a large population, whereas qualitative research lends itself very well to developing hypotheses and theories and to describing processes such as decision making or communication processes. Quantitative research generates factual, reliable outcome data that are usually generalizable to some larger populations, and qualitative research produces rich, detailed and valid process data based on the participant's, rather than the investigator's, perspectives and interpretations (1).

Quantitative research is usually deductive, relying on experimental and survey methods to test specific hypotheses based on general principles. It is strong in inductive reasoning, building and expanding theories concerning relationships among phenomena. In the actual practice of scientific research, theory and research interact through a never-ending cycle of deduction, induction, deduction, induction and so forth (2). By combining quantitative and qualitative methods, a degree of comprehensiveness may be achieved that neither approach, if used alone, can achieve. For example, to target populations of children who are not being immunized for common childhood infectious diseases, it is critical to quantify the existence of a low rate of immunization. However, to intervene to rectify the identified problem, it is important to explore why parents are not having their children vaccinated. Qualitative interviews are most appropriate for this purpose.
The nature of inquiry is similar in both quantitative and qualitative research, it is couched in the human desire to understand and explain behaviour and events, their components, antecedents, corollaries and consequences. If differences among researchers exist, it is not because they aspire to different ends, but because they have operationalized their methods for reaching those ends differently (3).

Even though both approaches are different from one another, one is not necessarily inferior to the other. However, qualitative research is often considered to be lacking scientific rigour. Unfortunately, the standard strategies used to enhance validity, reliability and objectivity in quantitative research are not always relevant to qualitative research. Fortunately, more attention is being paid to strategies to enhance the quality of the data and interpretations collected through qualitative research $(4,5)$. An example of a commonly used approach is triangulation, which refers to data collection in which evidence is deliberately sought from a wide range of different, independent sources and often by different means (for instance, comparing oral testimony with written records) (4).

The rigid demarcation between the two types of research has not encouraged interaction between the two camps. However, there are some good reasons for combining qualitative and quantitative research. First, a researcher may wish to explore an issue to understand what the relevant variables are or to develop hypotheses that can then be studied or tested in quantitative research. This way of combining the two approaches is also used in the development of scales or questionnaires. For example, qualitative techniques, such as observation, in-depth interviews or focus groups, can provide a description and understanding of a situation or behaviour. At their most basic, these techniques can be used simply to discover the most comprehensive terms or words to use in a subsequent survey questionnaire (6). Second, qualitative research also may follow quantitative research with the aim of explaining the quantitative results. For example, designing and evaluating an effective health campaign promoting influenza vaccinations faces multimethod challenges. To determine whether the campaign works so that the strategy can be effectively used again, it is not only important to identify how many people received shots, but also why and how they decided to get vaccinated (ie, linking process to outcome). Third, qualitative and quantitative research can be combined to enhance the validity of the results, much the same as in triangulation, but now using both quantitative and qualitative approaches, for their combined strength, rather than using one method to validate the result of the other. For example, overall validity would be enhanced through the use of a multimethods 
approach in instances where measuring the technical accuracy of a diagnostic or treatment intervention was important alongside an understanding of patient response to or acceptance of such a diagnostic test or treatment protocol (7).

Several barriers may prevent the further development of integrated research, such as lack of expertise, time and funding, mutual prejudices, and publication bias. If methodological integration is going to progress, a number of changes in the current environment are needed. These include acceptance and refinement of the underlying paradigms of qualitative and quantitative research, recognition by funding agencies of the need for both perspectives and a willingness to allocate sufficient resources, awareness by editorial boards of the importance of publishing multimethods research, training of researchers in both paradigms, encouragement of teamwork and promotion of mutual acceptance and respect by the adherents of each approach.

Given the multiple challenges facing health researchers today, broadening the horizons of health research to embrace the use and benefits of both quantitative and qualitative analysis is a methodological advance that must be supported and nurtured.

\section{REFERENCES}

1. Steckler A, McLeroy KR, Goodman RM, Bird ST, McGormick L. Toward integrating qualitative and quantitative methods, An introduction. Health Educ Q 1992;19:1-18.

2. Babbie E. The Practice of Social Research, 6th edn. Belmont: Wadsworth Publishing, 1992.

3. Dzurec LC, Abraham IL. The nature of inquiry: Linking quantitative and qualitative research. Adv Nurs Sci 1993;16:73-9.

4. Mays N, Pope C. Rigour and qualitative research. BM] 1995;311:109-12.

5. Krefting L. Rigour in qualitative research: The assessment of trustworthiness. Am J Occup Ther 1991;145:214-22.

6. Pope N, Mays C. Researching the parts other methods cannot reach: An introduction to qualitative methods in health and health services research. BMJ 1995;1311:42-5.

7. Daly J, MacDonald I, Willis E. Why don't you ask them? A qualitative research framework for investigating the diagnosis of cardiac normality. In: Daly J, MacDonald I, Willis E, eds. Researching Health Care: Designs, Dilemmas, Disciplines. London: Tavistock/Routledge, 1992:189-206.

Marja / Verhoef PhD, Ann L Casebeer PhD Department of Community Health Sciences Faculty of Medicine, The University of Calgary

Calgary, Alberta 


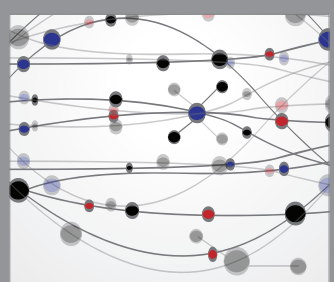

The Scientific World Journal
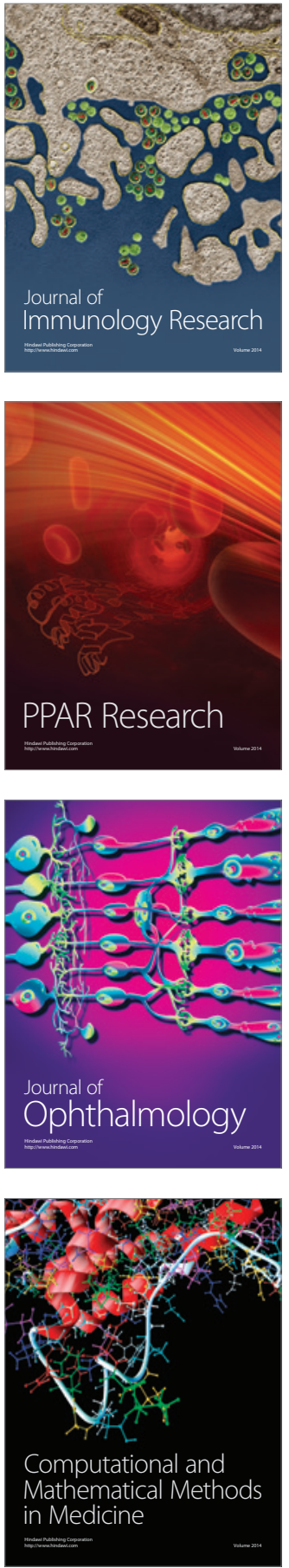

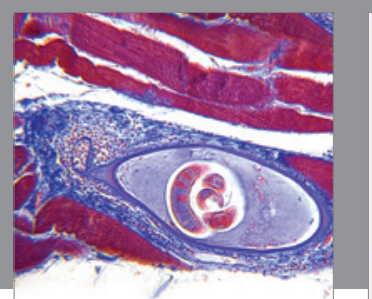

Gastroenterology Research and Practice

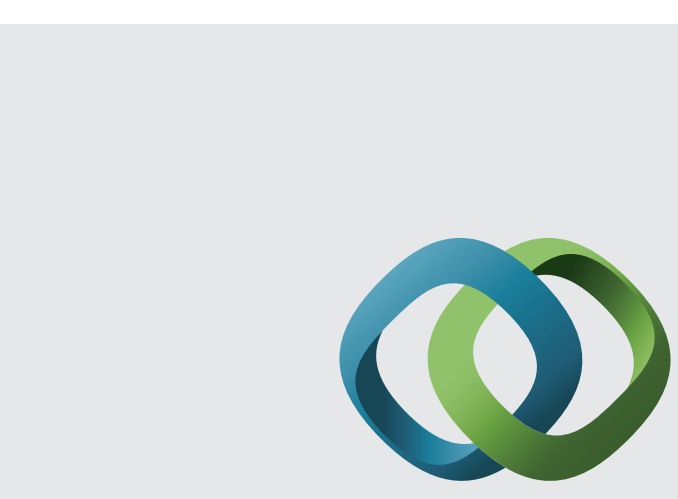

\section{Hindawi}

Submit your manuscripts at

http://www.hindawi.com
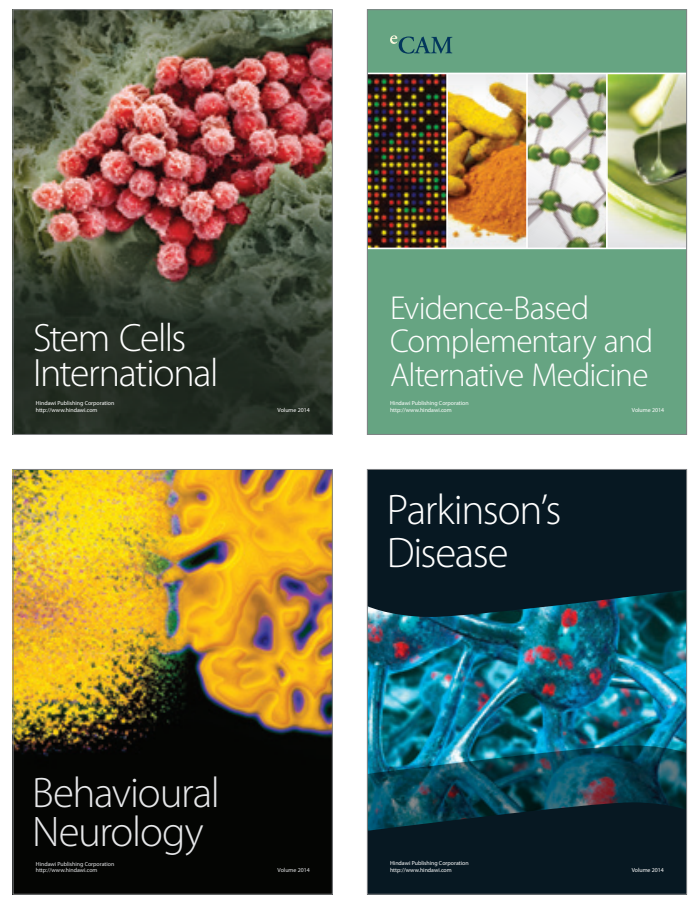
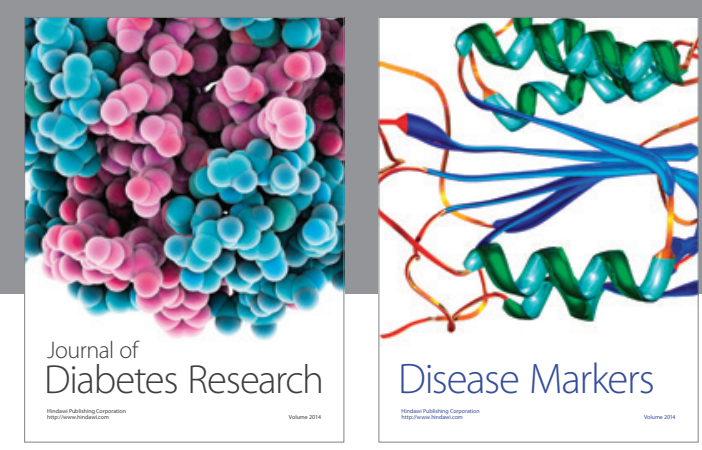

Disease Markers
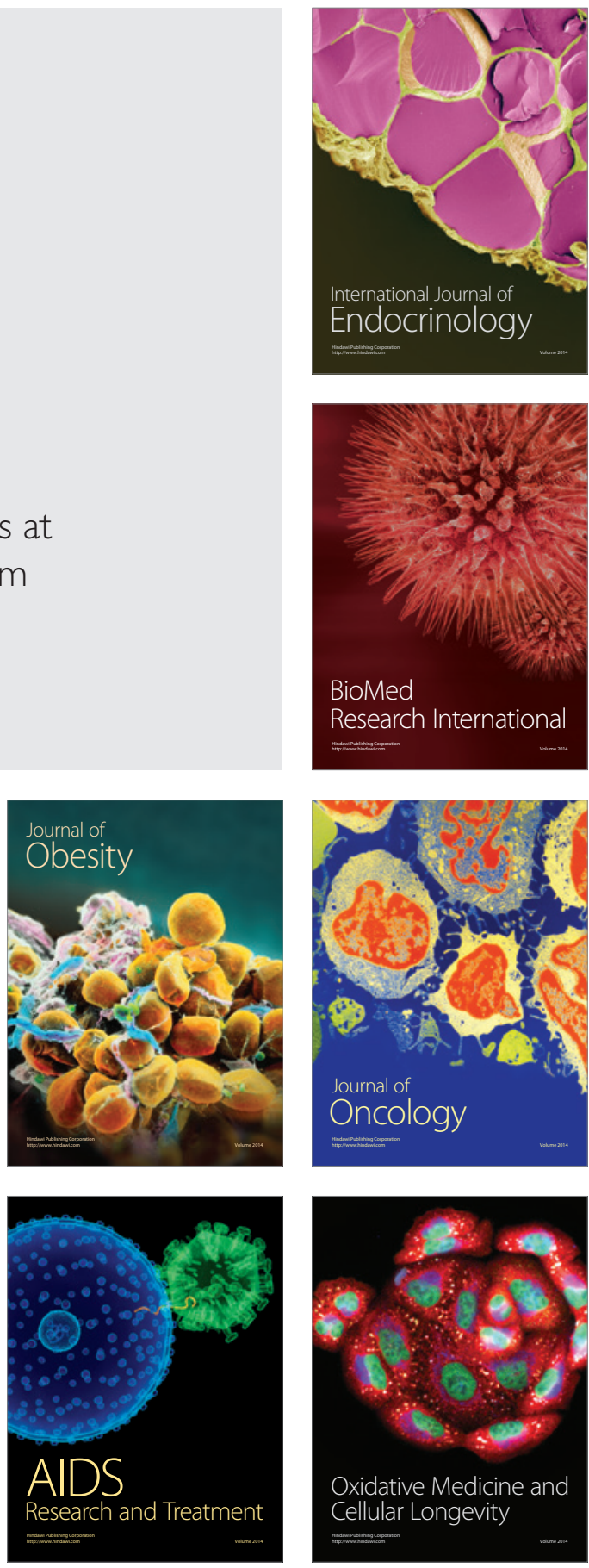